ISSN 0258-7122

Bangladesh J. Agril. Res. 38(3): 531-544, September 2013

\title{
STUDY OF HETEROSIS IN HEAT TOLERANT TOMATO (Solanum lycopersicum) DURING SUMMER
}

\author{
M. M. Alam PATWARY ${ }^{1}$, M. MIZANUR RAHMAN ${ }^{2}$, SHAHABUDDIN AHMAD $^{3}$ \\ M. A. KHALEQUE MIAH ${ }^{4}$ AND HAIMONTI BARUA ${ }^{5}$
}

\begin{abstract}
An experiment was conducted at the Vegetable Research Field of Olericulture Division, Horticulture Research Cente, Bangladesh Agricultural Research Institute (BARI), Gazipur during May to October 2008 to study heterosis using eight parents viz., $\mathrm{P}_{1}, \mathrm{P}_{2}, \mathrm{P}_{3}, \mathrm{P}_{4}, \mathrm{P}_{5}, \mathrm{P}_{6}, \mathrm{P}_{7}$, and $\mathrm{P}_{8}$. Most of the combinations showed better parent heterosis for earliness. Eight crosses showed positive heterosis for flower production. The highest heterotic effect for fruit set (\%) was found in the cross $\mathrm{P}_{6} \times \mathrm{P}_{7}(62.59 \%)$ followed by that in $\mathrm{P}_{7} \times \mathrm{P}_{8}(60.49 \%)$ and $\mathrm{P}_{1}$ $\times \mathrm{P}_{7}(40.00 \%)$. For fruits per plant, 8 crosses provided more than $15 \%$ heterosis over better parent. Considering fruit yield per plant, higher degree of heterosis was manifested by 24 hybrids over better parent ranging from 13.58 to 282.63 $\%$. Cross combination $\mathrm{P}_{4} \times \mathrm{P}_{7}$ showed the maximum significant positive heterosis followed by $\mathrm{P}_{6} \times \mathrm{P}_{7}(187.84 \%), \mathrm{P}_{4} \times \mathrm{P}_{8}(166.97 \%), \mathrm{P}_{3} \times \mathrm{P}_{7}(146.08$ $\%), \mathrm{P}_{3} \mathrm{xP}_{6}(103.92 \%)$, and $\mathrm{P}_{1} \mathrm{X}_{7}(100.45 \%)$ and the minimum in $\mathrm{P}_{4} \times \mathrm{P}_{6}$ $(13.58 \%)$. For viable pollens, $\mathrm{P}_{3} \times \mathrm{P}_{5}(20.56 \%)$ exhibited the highest positive heterosis. In case of shelf life, the highest heterosis was observed by the cross $\mathrm{P}_{3}$ $x \mathrm{P}_{6}(22.78 \%)$ followed by that in $\mathrm{P}_{4} \times \mathrm{P}_{6}(22.29 \%)$ and $\mathrm{P}_{2} \times \mathrm{P}_{6}(14.40 \%)$. For fruit flesh thickness, 12 hybrids exhibited more than $10 \%$ heterosis. Pollen tubes as well as viable pollens showed positive correlation with fruit set.
\end{abstract}

Keywords: Tomato, heterosis, heat tolerant tomato, summer.

\section{Introduction}

Heterosis is an important genetic phenomenon synonymous with hybrid vigour refers to the manifested superiority of the $F_{1}$ hybrid resulting from the cross of genetically dissimilar homozygous parents. The classical work of Shull and East during 1905-12 laid the foundations for exciting advances in exploitation of heterosis in crops (Taslim, 2006).

The best way to utilize heterosis in crop is to produce $F_{1}$ hybrids, which possess maximum heterozygosity.

The commercial exploitation of heterosis, however, was recorded first in 1930s with maize in USA (Ahmad, 2002). Now a days with many other hybrid

\footnotetext{
${ }^{1}$ Senior Scientific Officer, ARS, Bangladesh Agricultural Research Institute (BARI), Pahartali Chittagong, ${ }^{2 \& 4}$ Professor, Bangabandhu Sheikh Mujibur Rahman Agricultural University (BSMRU), Gazipur, ${ }^{3}$ Chief Scientific Officer, HRC, BARI, Gazipur and ${ }^{5}$ Scientific Officer, ARS, BARI, Pahartali, Chittagong, Bangladesh.
} 
crops, a lot of hybrid varieties of horticultural crops like tomato, brinjal, cabbage, cauliflower, cucumber, capsicum, broccoli, onion, and radish are frequently grown on large scale all over the world.

The exploitation of heterosis in the breeding and development of crop hybrids has made an enormous contribution to $20^{\text {th }}$ century agriculture, although the genetic basis of the phenomenon remains unclear (Rood et al., 1988; Sinha and Khanna, 1975). Geneticist and plant breeders described heterosis as the manifestation of greater vigour in height, leaf area, growth, dry matter accumulation, and yield in a $F_{1}$ hybrid in comparison with the parents (Allard, 1960; Hageman et al., 1967). $F_{1}$ hybrid tomatoes are one of the most leading vegetable crops all over the world. But these are absolutely for optimum growing season i.e., like winter season in Bangladesh. There is a bright scope of exploitation of heterosis under high temperature in Bangladesh as many tomato lines introduced from abroad are capable enough to produce a large number of small fruits. But a few attempts in this regard were done in the past. Therefore, the present investigation was an attempt to study the heterosis in tomato under high temperature conditions.

\section{Materials and Method}

The experiment was conducted at the experimental farm of Olericulture Division, Horticulture Research Centre (HRC), Bangladesh Agricultural Research Institute (BARI), Joydebpur, Gazipur during the month of May to October 2008. The average minimum and maximum temperature during the crop period was $25.22^{\circ} \mathrm{C}$ and $31.88^{\circ} \mathrm{C}$, respectively. A diallel cross of $8 \times 8$ excluding reciprocals were constructed from the eight heat tolerant parental lines viz., $\mathrm{P}_{1}, \mathrm{P}_{2}, \mathrm{P}_{3}, \mathrm{P}_{4}, \mathrm{P}_{5}, \mathrm{P}_{6}$, $\mathrm{P}_{7}$, and $\mathrm{P}_{8}$ collected from HRC, BARI, Gazipur. The basic seeds of the $8 \times 8$ halfdiallel mating were produced in the vegetable research field, HRC, BARI during October 2007 to March 2008. Seeds of the eight selfed parents and their $28 F_{1}$ hybrids were sown in seed bed on 5 May 2008. At the age of 30 days, seedlings were transplanted in the main experimental plots. The experiment was set up in a randomized complete block design (RCBD) with three replications. Thirty-six genotypes ( $28 \mathrm{~F}_{1} \mathrm{~s}+8$ parents) of tomato were considered as the 36 treatments of the experiment. The experimental plots were covered by transparent polythene with minimum interruption of photosynthesis. The polytunnel was 2.3 meter wide having two 1.0 meter wide bed with $30 \mathrm{~cm}$ drain in between, which serves as irrigation channel. Chemical fertilizers @ of $550 \mathrm{~kg}$ urea, $450 \mathrm{~kg}$ TSP, $250 \mathrm{~kg}$ MoP, $120 \mathrm{~kg}$ Gypsum, $2 \mathrm{~kg}$ boron, and 10 tons cowdung per hectare were applied. Half of the cowdung and the entire amount of TSP, Gypsum, and Boron were applied during final land preparation. The pits were prepared one week before planting. The remaining cowdung and $1 / 3$ of MoP were applied at that 
time. Top dressings were done in three equal installments at 10, 25, and 40 days after transplanting to apply the entire urea and rest $2 / 3$ of MoP. Weeding and mulching were done followed by top-dressing and irrigation at 15 days interval. Data on days to $50 \%$ flowering, flowers per cluster, viable pollens, fruit set (\%), fruited cluster/plant, fruits/plant, fruit weight $(\mathrm{g})$, fruit yield/plant $(\mathrm{g})$, viable pollens, fruit length $(\mathrm{cm})$, fruit diameter $(\mathrm{cm})$, branches/plant, plant height $(\mathrm{cm})$, seeds/fruit, 1000-seed weight, TSS, shelf life, locules/fruit, fruit firmness and fruit flesh thickness were recorded. All the recorded quantitative data were subjected to ANOVA. The significance of increase or decrease in $\mathrm{F}_{1}$ hybrids over their corresponding mid parent and better parent were tested by comparing their means with the help of appropriate standard error values in percentage. For estimation of heterosis in each character the mean values of the $28 \mathrm{~F}_{1} \mathrm{~s}$ have been compared with better parent (BP) for heterobeltiosis.

\section{Results and Discussion}

Analysis of variance for parents and crosses showed highly significant differences for all the characters studied (Table 1). The estimates of better parent heterosis observed in $\mathrm{F}_{1}$ generation are presented in Table 2 to 4.

\section{Days to 50\% flowering}

Negative heterosis over better parent was observed in case of days to $50 \%$ flowering showing earliness in the flowering and it was significant for almost all the cross combinations except one (Table 2). As the consumers prefer to eat tomato earlier, therefore, negative heterosis for this trait is preferable. This study is in accordance with the findings of Baishya et al. (2001) and Ahmad (2002) who also reported negative heterosis for this trait over the better parent in many of the crosses in their diallel progenies.

Table 1. Analysis of variance for genotypes (parents and crosses).

\begin{tabular}{|c|c|c|c|c|c|c|c|c|}
\hline \multirow{2}{*}{$\begin{array}{l}\text { Source of } \\
\text { variation }\end{array}$} & \multirow[t]{2}{*}{ d.f. } & \multicolumn{7}{|c|}{ Mean sum of square } \\
\hline & & $\begin{array}{c}\text { Days to } \\
50 \% \\
\text { flowering }\end{array}$ & $\begin{array}{c}\text { Flowers/ } \\
\text { cluster }\end{array}$ & Fruit set & $\begin{array}{c}\text { Fruit } \\
\text { cluster/ } \\
\text { plant }\end{array}$ & $\begin{array}{c}\text { Number } \\
\text { of fruits/ } \\
\text { plant }\end{array}$ & Fruit wt & Yield/ plant \\
\hline Blocks & 3 & 10.58 & 0.11 & 1.60 & 1.40 & 27.46 & 2.65 & 44334.76 \\
\hline $\begin{array}{l}\text { Genotypes } \\
(\text { Parents } \\
\left.\& \mathrm{~F}_{1} \mathrm{~s}\right)\end{array}$ & 35 & $31.25 * *$ & $4.20 * *$ & $194.59 * *$ & $15.04 * *$ & $128.58 * *$ & $1578.09 * *$ & $346133.11 * *$ \\
\hline Error & 105 & 1.00 & 0.27 & 6.05 & 0.47 & 3.31 & 6.02 & 8703.82 \\
\hline
\end{tabular}

** Significant at $1 \%$ level of probability. 
Table 1. Cont'd.

\begin{tabular}{l|c|c|c|c|c|c|c|c}
\hline Source of & d.f. & \multicolumn{8}{|c}{ Mean sum of square } \\
\cline { 2 - 9 } & $\begin{array}{c}\text { Fruit } \\
\text { length }\end{array}$ & $\begin{array}{c}\text { Fruit } \\
\text { diameter }\end{array}$ & $\begin{array}{c}\text { Branches/ } \\
\text { plant }\end{array}$ & $\begin{array}{c}\text { Plant } \\
\text { height }\end{array}$ & $\begin{array}{c}\text { Viable } \\
\text { pollens }\end{array}$ & $\begin{array}{l}\text { Seeds / } \\
\text { fruit }\end{array}$ & $\begin{array}{c}1000- \\
\text { seed wt }\end{array}$ \\
\hline $\begin{array}{c}\text { Blocks } \\
\text { Genotypes }\end{array}$ & 35 & $1.04^{* *}$ & $1.59^{* *}$ & $14.40^{* *}$ & $927.94^{* *}$ & $401.97^{* *}$ & $553.60^{* *}$ & $0.43^{* *}$ \\
$\begin{array}{c}\text { (Parents \& } \\
\left.\mathrm{F}_{1}, \mathrm{~s}\right)\end{array}$ & & & & & & & & \\
Error & 105 & 0.02 & 0.01 & 1.06 & 134.76 & 37.59 & 8.16 & 0.01 \\
\hline
\end{tabular}

** Significant at $1 \%$ level of probability.

Table 1. Cont'd.

\begin{tabular}{lc|c|c|c|c|c}
\hline $\begin{array}{l}\text { Source of } \\
\text { variation }\end{array}$ & d.f. & \multicolumn{5}{|c}{ Mean sum square } \\
\cline { 2 - 7 } & & TSS (\%) & Shelf life & Firmness & Locules/fruit & Thickness \\
\hline Replication & 2 & 0.04 & 0.14 & 0.02 & 0.04 & 0.02 \\
$\begin{array}{l}\text { Genotypes } \\
\text { (Parents and }\end{array}$ & 35 & $0.27^{* *}$ & $4.23^{* *}$ & $0.40^{* *}$ & $8.23^{* *}$ & $3.99^{* *}$ \\
$\begin{array}{l}\text { Crosses) } \\
\text { Error }\end{array}$ & 70 & & & & & \\
\hline
\end{tabular}

** Significant at $1 \%$ level of probability.

\section{Flowers per cluster}

Out of 28 crosses, only eight crosses showed positive heterosis for flower production and 20 showed significant negative heterosis over better parents. It indicated that flower production under high temperature have a minimum heterotic effect as most of the crosses showed significant negative heterosis (Table 2). Ahmad (2002) also found similar positive heterosis for this trait in a few cross combinations.

\section{Fruit set}

Significant heterosis either positive or negative was exhibited in relation to percent fruit set in almost all the cross combinations studied. Increased fruit set was observed in $50 \%$ crosses showing positive heterosis ranging from 0.08 to $62.59 \%$. The highest heterotic effect was found in the cross $\mathrm{P}_{6} \times \mathrm{P}_{7}(62.59 \%)$ followed by the cross $\mathrm{P}_{7} \times \mathrm{P}_{8}(60.49 \%), \mathrm{P}_{1} \times \mathrm{P}_{7}(40.00 \%), \mathrm{P}_{6} \times \mathrm{P}_{8}(39.33 \%)$ and $\mathrm{P}_{2} \times \mathrm{P}_{3}(31.80 \%)$ (Table 2). These findings of the present investigation are in conformity with the findings of Opena et al. (1987 a) and El-Ahmadi and Stevens (1979 b). 


\section{Fruit clusters per plant}

Like fruit set, 14 hybrids i.e. $50 \%$ of total crosses provided significantly heterosis positively, while the rest $50 \%$ hybrids exhibited significant negative heterosis except one. Positive heterosis for this character ranged from 1.20 to $79.52 \%$ over better parent in the crosses $\mathrm{P}_{2} \times \mathrm{P}_{7}$ and $\mathrm{P}_{6} \times \mathrm{P}_{7}$, respectively. More than $15 \%$ positive heterosis was manifested in 6 crosses (Table 2). Similar results were achieved by Ahmad (2002) while investigating heterosis for this trait. He observed more than $20 \%$ heterosis over better parent in 12 crosses.

\section{Fruits per plant}

Eleven crosses expressed significant positive heterosis for this trait. Out of them, more than $15 \%$ heterosis was manifested by eight crosses. Among them, hybrid $\mathrm{P}_{6} \times \mathrm{P}_{7}$ exhibited the highest (105.69\%) significant positive heterosis followed by $42.52 \%, 40.78 \%, 39.86 \%, 24.54 \%$, and $23.81 \%$ heterosis in the crosses $\mathrm{P}_{2}$ $x \mathrm{P}_{3}, \mathrm{P}_{4} \times \mathrm{P}_{8}, \mathrm{P}_{3} \times \mathrm{P}_{4}, \mathrm{P}_{3} \times \mathrm{P}_{6}$, and $\mathrm{P}_{3} \times \mathrm{P}_{7}$, respectively. The range of positive heterosis for this trait was 3.38 to $105.69 \%$ over better parent (Table 2). Maximum positive heterosis was observed in the cross $\mathrm{P}_{6} \times \mathrm{P}_{7}$ followed by that in $\mathrm{P}_{2} \times \mathrm{P}_{3}$ which were 105.69 and 42.52 percent, respectively. Similar findings of higher number of fruits per plant between several crosses were reported by Bhatt et al. (1999) and Sekar (2001).

\section{Fruit weight}

Fourteen $F_{1} s$ out of 28 showed positive heterosis for fruit weight indicated this trait can be improved through heterosis breeding. The rest 14 combinations expressed significant negative heterosis (Table 2).The highest positive heterosis over better parent was observed in the hybrid $\mathrm{P}_{4} \times \mathrm{P}_{5}(66.38 \%)$ followed by that of $\mathrm{P}_{5} \times \mathrm{P}_{6}(41.06 \%), \mathrm{P}_{6} \times \mathrm{P}_{7}(39.67 \%), \mathrm{P}_{1} \times \mathrm{P}_{8}(33.32 \%)$ and the lowest positive heterosis was recorded by the cross $\mathrm{P}_{4} \times \mathrm{P}_{6}(1.32 \%)$. Nine hybrids showed more than $15 \%$ positive heterosis for this trait. Heterosis for single fruit weight/plant under high temperature environments were reported by Fageria et al. (2001).

\section{Fruit yield per plant}

A high amount of heterosis was manifested by 24 hybrids over better parent ranging from 13.58 to $282.63 \%$ (Table 2). The cross combination $\mathrm{P}_{4} \times \mathrm{P}_{7}$ showed the maximum significant positive heterosis followed by $\mathrm{P}_{6} \times \mathrm{P}_{7}(187.84 \%), \mathrm{P}_{4} \mathrm{x}$ $\mathrm{P}_{8}(166.97 \%), \mathrm{P}_{3} \times \mathrm{P}_{7}(146.08 \%), \mathrm{P}_{3} \times \mathrm{P}_{6}(103.92 \%)$, and $\mathrm{P}_{1} \times \mathrm{P}_{7}(100.45 \%)$ (Table 2). Hassan et al. (2000) and Dharmatti et al. (2001) reported appreciable heterobeltiosis for yield per plant. Mahdy et al. (1990) and Ahmad (2002) also reported higher yield in the maximum cross combinations while studying heterosis in tomato. 
Table 2. Percent heterosis over better parent for days to $50 \%$ flowering, flowers/ cluster, fruit set (\%), fruit clusters/plant, fruits/ plant, fruit weight, yield/plant and viable pollens (\%).

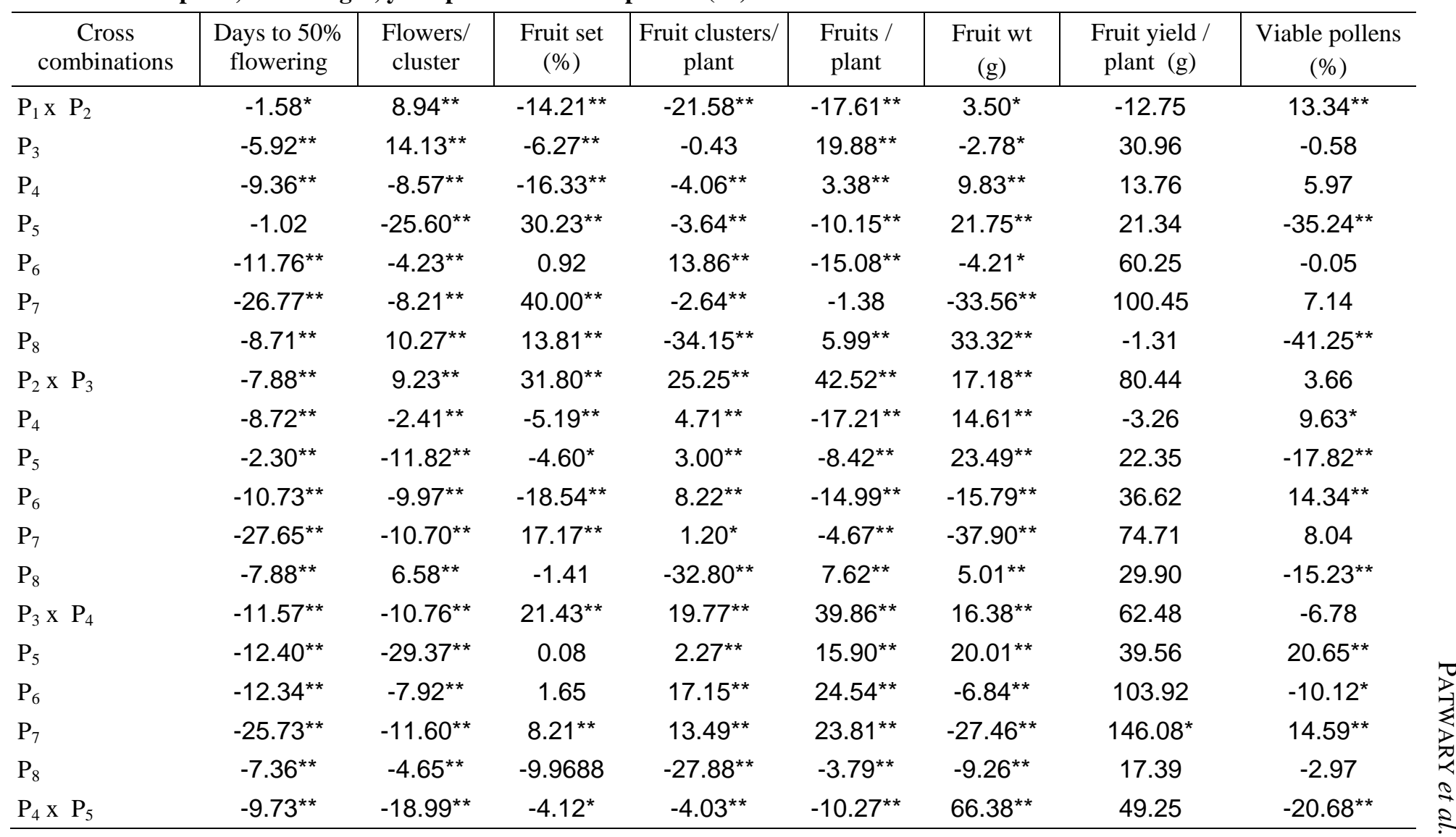


Table 2. Cont'd.

\begin{tabular}{|c|c|c|c|c|c|c|c|c|}
\hline $\mathrm{P}_{6}$ & $-8.71^{\star *}$ & $-13.16^{\star \star}$ & $-19.56^{\star \star}$ & $19.57^{\star \star}$ & $-27.76^{\star *}$ & 1.32 & 13.58 & -2.11 \\
\hline $\mathrm{P}_{7}$ & $-25.04^{\star *}$ & $-25.82^{* *}$ & 0.50 & $15.94^{\star *}$ & $-16.34^{\star *}$ & $-28.13^{\star \star}$ & $282.63^{\star *}$ & -4.91 \\
\hline $\mathrm{P}_{8}$ & $-11.25^{\star \star}$ & $7.72^{\star \star}$ & $-9.75^{\star \star}$ & $-24.37^{\star *}$ & $40.78^{\star \star}$ & $-5.75^{\star \star}$ & $166.97^{*}$ & $-15.14^{\star *}$ \\
\hline $\mathrm{P}_{7}$ & $-22.16^{\star *}$ & $-38.14^{\star \star}$ & $-11.76^{\star *}$ & $2.27^{\star \star}$ & $-2.86^{\star}$ & $-18.30^{* *}$ & 103.31 & $-10.71^{*}$ \\
\hline $\mathrm{P}_{8}$ & $-10.16^{\star *}$ & 0.26 & $-11.75^{\star *}$ & $-38.95^{\star *}$ & $-17.38^{* *}$ & $-19.56^{\star *}$ & -17.35 & $-24.95^{\star *}$ \\
\hline $\mathrm{P}_{7} \times \mathrm{P}_{8}$ & $-17.49^{* *}$ & $-15.08^{* *}$ & $60.49^{* *}$ & $-34.89^{* *}$ & $-33.28^{* *}$ & -45.08 & 78.54 & 0.28 \\
\hline SE & 0.71 & 0.37 & 1.74 & 0.48 & 1.29 & 1.74 & 65.97 & 4.34 \\
\hline CD (5\%) & 1.44 & 0.75 & 3.54 & 0.99 & 2.62 & 3.53 & 134.12 & 8.81 \\
\hline CD (1\%) & 1.93 & 1.01 & 4.75 & 1.32 & 3.52 & 4.74 & 180.16 & 11.84 \\
\hline
\end{tabular}




\section{Viable pollens}

For viable pollen grains, $11 \mathrm{~F}_{1} \mathrm{~s}$ out of 28 showed better parent heterosis positively. The cross combination $\mathrm{P}_{3} \times \mathrm{P}_{5}(20.65 \%)$ exhibited the maximum positive heterosis followed by $\mathrm{P}_{3} \times \mathrm{P}_{7}(14.59 \%)$ and $\mathrm{P}_{2} \times \mathrm{P}_{6}(14.34 \%)$ and the lowest positive heterosis was recorded from $\mathrm{P}_{7} \times \mathrm{P}_{8}(0.28 \%)$ (Table 2). Ahmad (2002) also found similar heterosis for this trait but the degree of positive heterosis was several folds higher. This might be due to seasonal variations as well as genetical differences among the germplasm under studied.

\section{Fruit length}

Eighteen crosses showed positive heterosis for this trait over better parents ranged from $0.88 \%\left(\mathrm{P}_{2} \times \mathrm{P}_{5}\right)$ to $14.12 \%\left(\mathrm{P}_{4} \times \mathrm{P}_{6}\right)$ (Table 3). Sharma et al. (2001) reported hybrid vigour over better parent for fruit length.

\section{Fruit diameter $(\mathbf{c m})$}

Fifteen hybrids provided positive heterosis of which 14 hybrids were significant for fruit diameter except one, while 13 hybrids showed significant negative heterosis (Table 3 ). The range of positive heterosis varied from $0.16 \%$ to 21.22 $\%$ by the hybrids $\mathrm{P}_{6} \times \mathrm{P}_{7}$ and $\mathrm{P}_{5} \times \mathrm{P}_{6}$, respectively This result coincides with the findings of Alvarez (1985) and Susic (1998).

\section{Branches per plant}

It is evident from the Table 3 that 19 hybrids performed positive heterosis indicating the number of branches per plant can be increased significantly through heterosis breeding. The highest heterosis was achieved by the hybrid $\mathrm{P}_{3}$ $\times \mathrm{P}_{7}(29.37 \%)$. Singh and Singh (1993) and Ahmad (2002) reported heterosis for the number of branches per plant over the better parent in the maximum hybrids in tomato.

\section{Plant height}

In case of plant height at last harvest, though 18 cross combinations out of 28 provided positive heterosis over better parent, among them, only 4 crosses showed significant positive heterosis (Table 3). The heterobeltiotic effects ranged from $-21.19 \%$ to $36.72 \%$. Maximum significant positive heterosis was evident in the cross $\mathrm{P}_{2} \times \mathrm{P}_{5}(36.72 \%)$ followed by that in $\mathrm{P}_{1} \times \mathrm{P}_{7}(26.79 \%)$. Heterosis in relation to plant height under high temperature was also manifested by Fageria $e t$ al. (2001). 
Table 3. Percent heterosis over better parent for fruit length, fruit diameter, branches/ plant, plant height, seeds/ fruit, 1000-seed weight, TSS (\%), and shelf life.

\begin{tabular}{|c|c|c|c|c|c|c|c|c|}
\hline Cross combinations & $\begin{array}{l}\text { Fruit length } \\
\quad(\mathrm{cm})\end{array}$ & $\begin{array}{l}\text { Fruit } \\
\text { diameter } \\
(\mathrm{cm})\end{array}$ & $\begin{array}{l}\text { Branches/ } \\
\text { plant }\end{array}$ & $\begin{array}{l}\text { Plant height } \\
(\mathrm{cm})\end{array}$ & $\begin{array}{l}\text { Seeds/ } \\
\text { fruit }\end{array}$ & $\begin{array}{c}\text { 1000-seed wt } \\
(\mathrm{g})\end{array}$ & $\begin{array}{l}\text { TSS } \\
(\%)\end{array}$ & $\begin{array}{c}\text { Shelf life } \\
\text { (days) }\end{array}$ \\
\hline $\mathrm{P}_{3}$ & $1.35^{\star \star}$ & $-4.04^{* *}$ & $5.61^{* *}$ & 7.05 & -1.10 & $7.12^{\star *}$ & $2.39^{* *}$ & $6.56^{\star \star}$ \\
\hline $\mathrm{P}_{4}$ & $4.50^{\star \star}$ & $0.34^{\star *}$ & $-22.80^{\star *}$ & -4.28 & $65.50^{* *}$ & $6.52^{\star \star}$ & $1.36^{\star *}$ & $5.32^{\star \star}$ \\
\hline $\mathrm{P}_{6}$ & $8.35^{\star \star}$ & $5.28^{\star *}$ & $4.59^{\star *}$ & -1.17 & $53.08^{\star *}$ & $9.96^{\star \star}$ & $6.82^{* *}$ & $7.00^{\star *}$ \\
\hline $\mathrm{P}_{7}$ & $6.76^{\star \star}$ & $-18.82^{\star \star}$ & $17.13^{* \star}$ & $26.79^{\star *}$ & $-63.53^{\star *}$ & $-11.74^{\star \star}$ & $8.21^{* *}$ & $-3.44^{\star *}$ \\
\hline $\mathrm{P}_{8}$ & $-4.49^{\star \star}$ & $-1.31^{* *}$ & $-32.25^{\star \star}$ & -21.19 & $29.29^{\star *}$ & $-6.60^{\star \star}$ & $4.41^{\star *}$ & $-12.32^{\star *}$ \\
\hline $\mathrm{P}_{2} \times \mathrm{P}_{3}$ & $-0.95^{\star \star}$ & $1.03^{\star *}$ & $20.03^{\star *}$ & 4.71 & $3.40^{*}$ & $19.38^{\star *}$ & $-2.53^{\star *}$ & $-2.65^{\star *}$ \\
\hline $\mathrm{P}_{7}$ & $5.75^{\star \star}$ & $-26.63^{\star *}$ & $10.49^{* \star}$ & 11.69 & $-68.54^{\star \star}$ & $-8.30^{\star \star}$ & $7.62^{\star \star}$ & $-3.69^{\star \star}$ \\
\hline $\mathrm{P}_{8}$ & $-5.94^{\star *}$ & $-4.56^{\star *}$ & $-30.32^{* *}$ & -3.52 & $-6.36^{* *}$ & $9.34^{* *}$ & $8.24^{* *}$ & $3.60^{\star \star}$ \\
\hline $\mathrm{P}_{3} \times \mathrm{P}_{4}$ & $5.86^{\star *}$ & $8.78^{\star *}$ & $16.00^{\star *}$ & 2.52 & $-34.38^{\star *}$ & $14.30^{\star *}$ & $-3.45^{\star *}$ & $2.32^{* *}$ \\
\hline $\mathrm{P}_{5}$ & $-5.78^{\star \star}$ & $0.23^{\star *}$ & $11.74^{\star \star}$ & 6.54 & $36.97^{\star *}$ & $0.30^{\star *}$ & $2.23^{\star *}$ & $-2.64^{\star *}$ \\
\hline $\mathrm{P}_{6}$ & $3.58^{\star *}$ & $7.15^{\star \star}$ & $4.09^{\star *}$ & 8.07 & $66.17^{\star *}$ & $35.29^{\star *}$ & $4.02^{* \star}$ & $22.78^{* *}$ \\
\hline
\end{tabular}




\begin{tabular}{|c|c|c|c|c|c|c|c|c|}
\hline $\mathrm{P}_{7}$ & $3.25^{\star *}$ & $-20.57^{\star *}$ & $29.37^{\star *}$ & 8.02 & $-36.30^{* *}$ & $2.21^{\star *}$ & $6.54^{* *}$ & $2.86^{\star *}$ \\
\hline $\mathrm{P}_{8}$ & $-3.05^{\star *}$ & $-2.58^{\star *}$ & $-29.70^{* *}$ & -10.15 & $-48.08^{* *}$ & $18.75^{\star *}$ & $-12.51^{* *}$ & $-2.91^{* *}$ \\
\hline $\mathrm{P}_{4} \times \mathrm{P}_{5}$ & $1.58^{* *}$ & $13.09^{* *}$ & $1.61^{*}$ & 0.95 & $19.49^{\star *}$ & $-10.33^{* *}$ & $-3.38^{* *}$ & $2.53^{\star *}$ \\
\hline $\mathrm{P}_{6}$ & $14.12^{\star *}$ & $9.35^{\star *}$ & $5.10^{* *}$ & 8.14 & $68.41^{* *}$ & $14.29^{\star *}$ & $11.49^{* *}$ & $22.29^{* \star}$ \\
\hline $\mathrm{P}_{7}$ & $5.07^{* *}$ & $-19.99^{* *}$ & $8.95^{\star *}$ & 7.63 & $-58.06^{\star *}$ & $-16.53^{* *}$ & $3.66^{* *}$ & $-2.35^{\star *}$ \\
\hline $\mathrm{P}_{8}$ & $3.75^{\star *}$ & $3.15^{\star *}$ & $-46.59^{* *}$ & $-20.51^{*}$ & $-59.24^{\star *}$ & $4.17^{\star \star}$ & $-8.32^{* *}$ & $-3.47^{* *}$ \\
\hline $\mathrm{P}_{5} \times \mathrm{P}_{6}$ & $-3.45^{\star *}$ & $21.22^{\star *}$ & $14.75^{\star \star}$ & 3.95 & $44.76^{* *}$ & $-23.40^{* \star}$ & $4.80^{* *}$ & -0.89 \\
\hline $\mathrm{P}_{7}$ & $-3.65^{\star *}$ & $-9.41^{* *}$ & $22.66^{* *}$ & $19.32^{*}$ & $-26.17^{* *}$ & $0.91^{* *}$ & $-17.11^{* *}$ & 0.02 \\
\hline $\mathrm{P}_{8}$ & $-7.58^{\star *}$ & $-18.43^{\star *}$ & $-34.94^{* *}$ & $-17.78^{\star}$ & $117.55^{\star *}$ & $-3.04^{\star *}$ & $2.30^{\star *}$ & $-12.13^{\star \star}$ \\
\hline $\mathrm{P}_{6} \times \mathrm{P}_{7}$ & $13.92^{\star *}$ & 0.16 & $2.24^{* *}$ & -4.78 & $39.05^{* *}$ & $12.65^{\star \star}$ & $2.20^{* *}$ & 0.29 \\
\hline $\mathrm{P}_{8}$ & $-3.78^{* *}$ & $-4.80^{* *}$ & $-25.12^{\star *}$ & -5.37 & $21.33^{* *}$ & $-7.64^{\star *}$ & $-11.87^{\star *}$ & $-3.40^{* *}$ \\
\hline $\mathrm{P}_{7} \times \mathrm{P}_{8}$ & $-7.30^{\star *}$ & $-29.13^{* *}$ & $-21.38^{\star *}$ & $-22.92^{* *}$ & $6.81^{* *}$ & $-11.46^{* *}$ & $-8.53^{* *}$ & $2.50^{* *}$ \\
\hline SE & 0.09 & 0.08 & 0.73 & 8.21 & 2.02 & 0.06 & 0.09 & 0.48 \\
\hline CD (5\%) & 0.18 & 0.17 & 1.48 & 16.69 & 4.11 & 0.13 & 0.19 & 0.97 \\
\hline CD (1\%) & 0.24 & 0.23 & 1.99 & 22.42 & 5.52 & 0.17 & 0.26 & 1.30 \\
\hline
\end{tabular}

* Significant at $5 \%$ level of probability, ** Significant at $1 \%$ level of probability 


\section{Seeds per fruit}

Significant positive heterosis were manifested by 16 crosses varying from 3.40 to $117.55 \%$ (Table 3 ). The highest heterotic value was achieved by the hybrid $P_{5} \times$ $\mathrm{P}_{8}(117.55 \%)$ followed by the crosses $\mathrm{P}_{4} \times \mathrm{P}_{6}(68.41 \%), \mathrm{P}_{1} \times \mathrm{P}_{5}(67.56 \%)$, and $\mathrm{P}_{1}$ $x \mathrm{P}_{4}(65.50 \%)$ and the lowest positive heterosis was provided by the hybrid $\mathrm{P}_{2} \mathrm{x}$ $\mathrm{P}_{3}(3.40 \%)$. El-Ahmadi and Stevens (1979b) and Ahmad (2002) reported higher degree of heterosis for this trait.

\section{0-seed weight}

Highly significant positive heterosis regarding 1000-seed weight was observed by 19 hybrids indicating seed quality can be improved through hybridization (Table 3). This result is in accordance with the findings of Subburamu et al. (1999).

\section{Total soluble solids}

Out of 28 crosses, positive highly significant heterosis regarding total soluble solids was found in 18 hybrids. The highest positive heterosis $(11.49 \%)$ was observed by the cross $\mathrm{P}_{4} \times \mathrm{P}_{6}(11.49 \%)$ followed by that in $\mathrm{P}_{1} \times \mathrm{P}_{2}(10.25 \%)$ and the lowest positive heterosis was recorded in the cross $\mathrm{P}_{1} \times \mathrm{P}_{4}(1.36 \%)$ (Table 3). This result is in accordance with the findings of Bhatt et al. (1999).

\section{Shelf life}

Shelf life is one of the most important physical attributes for tomato as increased shelf life prevents the post harvest loss with a significant manner. Here, about $50 \%$ cross combinations exhibited positive high significant heterosis with a few exceptions (Table 3). High heterosis in terms of shelf life was also revealed by Premalakshmi (2002).

\section{Locules per fruit}

Only five cross combinations out of 28 showed significant positive heterobeltiosis (Table 4). Negative heterosis was also demonstrated in most of the cross combinations by Taslim (2006). Kurian and Peter (2001) also identified heterotic hybrids for locule number in tomato.

\section{Fruit firmness}

Appreciable positive heterosis ranging from $4.61 \%$ to $33.33 \%$ was recorded by several hybrids over the best parental lines for this character (Table 4). Premalakshmi (2002) mentioned high heterosis over better parents with other qualitative characters. 


\section{Fruit flesh thickness}

Highly significant heterosis was manifested by majority of the cross combinations towards positive heterosis over better parental lines (Table 4). The 12 hybrids exhibited more than $10 \%$ heterosis indicating improvement on fruit quality like flesh thickness is possible with a significant manner through heterosis breeding. Positive heterosis in relation to flesh thickness was also observed by the several workers such as Dhaliwal et al. (1999), Sharma et al. (2001) and Makesh et al. (2002).

Table 4. Percent heterosis over better parent in summer season for locules per fruit, fruit firmness and flesh thickness.

\begin{tabular}{|c|c|c|c|}
\hline Cross combinations & Locules/fruit & Fruit firmness & $\begin{array}{l}\text { Flesh thickness } \\
(\mathrm{mm})\end{array}$ \\
\hline $\mathrm{P}_{1} \times \mathrm{P}_{2}$ & $-20.01 * *$ & $15.98 * *$ & $0.60 * *$ \\
\hline $\mathrm{P}_{3}$ & $-18.41 * *$ & $33.33 * *$ & $-13.38 * *$ \\
\hline $\mathrm{P}_{4}$ & $-30.07 * *$ & $23.10 * *$ & $3.20 * *$ \\
\hline $\mathrm{P}_{5}$ & $-46.81 * *$ & $5.86 * *$ & $-6.09 * *$ \\
\hline $\mathrm{P}_{6}$ & $-14.57 * *$ & $14.27 * *$ & $9.24 * *$ \\
\hline $\mathrm{P}_{7}$ & $37.88 * *$ & $19.26 * *$ & $0.82 * *$ \\
\hline $\mathrm{P}_{8}$ & $-22.38 * *$ & $15.99 * *$ & $-6.31 * *$ \\
\hline $\mathrm{P}_{2} \times \mathrm{P}_{3}$ & $27.83 * *$ & $-1.54 * *$ & $6.91 * *$ \\
\hline $\mathrm{P}_{4}$ & $-2.37 * *$ & $-3.84 * *$ & $12.86 * *$ \\
\hline $\mathrm{P}_{5}$ & $-36.57 * *$ & $-20.43 * *$ & $7.05 * *$ \\
\hline $\mathrm{P}_{6}$ & $7.33 * *$ & $-8.40 * *$ & $36.23 * *$ \\
\hline $\mathrm{P}_{7}$ & $-57.07 * *$ & $8.29 * *$ & $49.81 * *$ \\
\hline $\mathrm{P}_{8}$ & $29.17 * *$ & $-7.39 * *$ & $19.69 * *$ \\
\hline $\mathrm{P}_{3} \times \mathrm{P}_{4}$ & $-2.51 * *$ & $-16.83 * *$ & $9.55^{* *}$ \\
\hline $\mathrm{P}_{5}$ & $-41.00 * *$ & $-14.29 * *$ & $5.76 * *$ \\
\hline $\mathrm{P}_{6}$ & $-20.33 * *$ & $-14.67 * *$ & $21.52 * *$ \\
\hline $\mathrm{P}_{7}$ & $-19.51 * *$ & $11.70 * *$ & $21.00 * *$ \\
\hline $\mathrm{P}_{8}$ & 0.00 & $-35.62 * *$ & $13.07 * *$ \\
\hline $\mathrm{P}_{4} \times \mathrm{P}_{5}$ & $-43.36 * *$ & $-4.29 * *$ & $13.02 * *$ \\
\hline $\mathrm{P}_{6}$ & $-33.33 * *$ & $-4.67 * *$ & $-12.07 * *$ \\
\hline $\mathrm{P}_{7}$ & $-39.75 * *$ & $6.60 * *$ & $-23.52 * *$ \\
\hline $\mathrm{P}_{8}$ & $-16.32 * *$ & 0.00 & $-39.48 * *$ \\
\hline $\mathrm{P}_{5} \times \mathrm{P}_{6}$ & $-12.09 * *$ & $-13.20 * *$ & $16.06 * *$ \\
\hline $\mathrm{P}_{7}$ & $-4.55 * *$ & $4.61 * *$ & $23.80 * *$ \\
\hline $\mathrm{P}_{8}$ & $-55.40 * *$ & $-33.33 * *$ & $-4.51 * *$ \\
\hline $\mathrm{P}_{6} \mathrm{X} \mathrm{P}_{7}$ & $5.96 * *$ & $13.47 * *$ & $-1.96 * *$ \\
\hline $\mathrm{P}_{8}$ & $-11.00 * *$ & $-1.73 * *$ & $65.25 * *$ \\
\hline $\mathrm{P}_{7} \times \mathrm{P}_{8}$ & $-52.39 * *$ & $-15.73 * *$ & $88.75 * *$ \\
\hline $\mathrm{SE}$ & 0.13 & 0.06 & 0.15 \\
\hline $\mathrm{CD}(5 \%)$ & 0.27 & 0.13 & 0.31 \\
\hline $\mathrm{CD}(1 \%)$ & 0.37 & 0.17 & 0.42 \\
\hline
\end{tabular}

* Significant at $5 \%$ level of probability, ${ }^{* *}$ Significant at $1 \%$ level of probability. 
Based upon the above results and discussion, it is clear that appreciable heterosis towards desirable direction were manifested by all the characters studied. In addition, it can be concluded that the improvement of yield and yield contributing characters including qualitative attributes in tomato can be accomplished through heterosis breeding. The presence of high heterosis indicated genetic diversity within the parental lines. Therefore, the hybrids those are capable enough to express better yield potential with acceptable qualitative performances as compared to the existing varieties could be used for commercial utilization.

\section{References}

Ahmad, S. 2002. Genetics of fruit set and related traits in tomato under hot humid conditions. Ph D Thesis. Bangabandhu Sheikh Mujibur Rahman Agricultural University, Salna, Gazipur, Pp. 1-236.

Alvarez, M. 1985. Evaluation of tomato hybrids in summer. II. Heterosis for morphological characteristics and fruit weight. Cultivars-Tropicals 7(1): 37-45.

Allard, R. W. 1960. Principles of plant breeding. John Wiley and Song, Inc. New York. Pp. 189-222.

Baishya, K. C., M. M. Syamal and K. P. Singh. 2001. Heterosis studies in tomato (Lycopersicon esculuntum Mill.). Veg. Sci. 28(2): 168-169.

Bhatt, R.P., V. R. Biswas, K. Narendra and N. Kumar.1999. Studies of heterosis for certain characters in tomato (Lycopersicon lycopersicum L.) under mid hill condition. Progressive Hort. 31(1-2): 41-43.

Dhaliwal, M.S., S. Surjan, B. S. Badha, D. S. Cheema and S. Singh. 1999. Diallel analysis for total soluble solids content, pericarp thickness and locule number in tomato. Veg. Sci. 26 (2): 120-122.

Dharmatti, P.R., B. B. Madalgeri, I. M. Mannikeri, R. V. Patil and G. Patil. 2001. Genetic divergence studies in summer tomatoes. Karnataka J. Agric. Sci. 14(2): 407411.

El-Ahmadi, A. B. and M. A. Stevens. 1979b. Genetics of high temperature fruit set in the tomato. J. Amer. Soc. Hort. Sci. 104: 691-696.

El-Mahdy, I., E-Metwally, G. El-Fadly and A.Y. Mazrouh. 1990. Inheritance of yield and fruit setting quality of some tomato crosses grown under heat stress conditions in Egypt. J. Agric. Res. Tanta Univ. 16 (3): 517-526.

Fageria, M. S., U. K. Kohli and R. S. Dhaka. 2001. Studies on heterobeltiosis for fruit yield and yield attributing traits in tomato. Haryana J. Hort. Sci. 30(1-2): 131-133.

Hageman, R. H., E. R. Leng and J. W. Dudley. 1967. A biochemical approach to corn breeding. Adv. Agron. 19: 45-86.

Hassan, A. A., S. E. S. Moustafa, A. K. Abdel and A. A. Mohammad. 2000. Development and release of some new tomato (Lycopersicon esculuntum Mill.) hybrids. Egyptian J. Hort. 27(2): 210-218. 
Kurian, A. and K. V. Peter. 2001. Heterosis for quality traits in tomato. J. Tropical Agric. 39(1): 5-8.

Makesh, S, M. Pudan, S. Ashok and M. R. Banu. 2002. Heterosis studies for yield and quality in tomato. Advances in Plant Sci. 15(2): 597-601.

Opena, R. T., S. Shanmugasundaram, J. Y. Yoon and G. C. F. Fernandez. 1987a. Crop improvement program to promote vegetable production in the tropics. In: Breeding of horticultural Crops. Eds. W. N. Chang, R. T. Opena and J. B. Peterson. Food and Fertilizer Technology Center, Taipei, Taiwan. Pp. 1-17.

Premalakshmi, V., T. Thangaraj and T. Arumugam. 2002. Hybrid vigour for yield and shelf life in tomato(Lycopersicon esculuntum Mill.). South Indian Hort. 50(4-6): 360-369.

Rood, S. B., R. I. Buzzel and McDonald, M. D. 1988. Influence of temperature on heterosis in maize seedling growth. Crop Sci. 28: 283-286.

Sekar, K. 2001. Heterosis for yield and yield components of tomato (Lycopersicon esculuntum Mill.). Advances in Hort. and Forestry 8: 95-102.

Sharma, D. K., D. R. Chaudhary and D. P. Pandey. 2001. Studies on hybrid vogour in tomato (Lycopersicon esculuntum Mill.). Haryana J. Hort. Sci. 30(3): 236-238.

Singh, R. K. and V. K. Singh. 1993. Heterosis Breeding in tomato (Lycopersicon esculuntum Mill.). Ann. Agric. Res. 14(4): 416-420.

Sinha, S. K. and R. Khanna. 1975. Physiological, biochemical and genetic basis of heterosis. Advances in Agronomy 27:123-174.

Subburamu K., M. Jayapragasam and V. Thandapani.1999. Heterosis for seed and seedling characters in tomato (Solanum lycopersicum). Seed-Res. 26(2): 187-190.

Susic, Z. 1998. Effects of parental germplasm on inheriting the characteristics of $F_{1}$ generation of tomato hybrids. Rev. Res. Work. The faculty Agric. Belgrade. 43(2): 63-73.

Taslim, A. 2006. Combining ability and heterosis in tomato (Solanum lycopersicum.). M. S. Thesis. Bangabandhu Sheikh Mujibur Rahman Agricultural University, Salna, Gazipur. P. 85. 